\title{
Editorial
}

\section{Technical to operations: The diversity of revenue management}

\author{
Journal of Revenue and Pricing Management (2014) 13, 271-272. doi:10.1057/rpm.2014.23
}

This issue encompasses the diversity and maturity of revenue management (RM) as an academic subject addressing the operations management questions of implementation, technical developments in applied operations research and media studies. Too begin with, Walczak and Kambour addressed fare families with price-sensitive demand. Typically, fare families correspond to product categories that differ by relatively more important features such as refundability or baggage fees, while products within a family may be differentiated to a lesser degree. Organizing product offerings in this way is thought to help with better management of price-sensitive demand. The authors proposed a way to accommodate fare families by building upon the traditional yield demand concept. Bhatta and colleagues examined the media and public message about the payas-you-weigh (PAYW) pricing model of an air ticket. The PAYW pricing model of an air ticket envisages passenger's body weight as a major determinant (at least in part) of a fare. As opposed to the commonly held view, the model argues that it is not a seat but weight is a product that an airline sells in a flight. As a result, lighter passengers should get a discount, while heavier passengers should pay more according to the model. The article addresses how the media portrayed such a model, focusing on the concept of obesity and fat taxes. Others portrayed the concept in relation to discrimination and humanitarian aspects, transactions costs and practical problems of implementing the model, and so forth.

Talon-Ballestero and colleagues propose a new model to assess the implementation of RM for the hotel industry called MERMI. The model comes about because in today's increasingly competitive business world, new philosophies and ideas are required. Although RM is well developed in the airline industry, it is underresearched in the hospitality sector. A detailed analysis of the results of the Delphi study showed that the experts ranked the categories comprising the ideal model by order of importance as follows: culture, forecasting, segmentation, pricing, distribution channel analysis, analysis of the competition, calculating and updating reservation and sales limits, evaluation, and budgeting. The significance of the study concluded a more logical structure, in which stages are listed chronologically and activities are organized to be consistent with the objectives of their respective stage. In addition, the enormous importance attached to the development of RM culture, based on its acceptance by customers and employees through information and training, is essential to this process. And finally, the inclusion of forecasting as a stage of the process that encompasses market and supply-side analysis.

Lanquepin-Chesnais delves into the world of profit maximization version of the dynamic economical problem, where we charge a cost per price change. This model makes an explicit link between the number of price changes and the cost of changing prices. The author's heuristic ponders that if prices and orders have to be determined jointly in a first step, the pricing could be in practice 'improved' in a second step to tackle adverse effects of dynamic pricing. Such a property makes sense only when the 
problem is uncapacitated. Conversely, the pricing is determined not only by orders but also by resource limitations. Further research would be to consider how cost of price adjustments interact with capacity.

Given the high competition and the inherent variability of most markets, airlines learnt to quickly react to the changes in their passenger Origin-Destination flows, either by changing route capacities and frequencies, or by implementing RM techniques, such as changes in pricing and inventory control. Oancea argues that RM decisions cannot be based solely on recorded transaction data, and experienced revenue managers are aware of the risks of relying too much on booking figures. The main risks are that the analysis of the quality of RM actions based on such data can sometimes be very difficult because of demand variability and other factors involved that the reliability of detruncated booking data as a proxy of historical demand can be very poor. Concluding the design of such decision support tools needs to incorporate more external data and reflect the thought process of decision making.

Ian Yeoman

Editor 